\title{
Efeito do fornecimento de ração complementada com semente de linhaça sobre os macronutrientes e colesterol em tecidos de camaróes da Malásia (Macrobrachium rosenbergii) \\ Effect of Supplying Food Complemented with Linseed on the Chemical Composition of Malaysian Shrimp (Macrobrachium rosenbergii)
}

\author{
Fabiana Lindenberg dos SANTOS ${ }^{1}$, Vilma Blondet de AZEREDO ${ }^{1 *}$, Antonio Sérgio Aymoré MARTINS ${ }^{2}$
}

\begin{abstract}
Resumo
Este estudo teve como objetivo avaliar a composição centesimal dos camarões alimentados com dieta complementada com semente de linhaça. Foram utilizados 800 camarões pós-larvas, divididos em 2 tratamentos: 1) grupo teste à base de caseína complementada com linhaça; e 2) grupo caseína. Os valores médios de umidade $(79,8 \pm 0,82 ; 78,9 \pm 1,68)$, cinzas $(0,62 \pm 0,07 ; 0,68 \pm 0,2)$, carboidratos $(2,63 \pm 0,13 ; 1,58 \pm 0,7)$ e proteínas $(15,6 \pm 1,02 ; 16,0 \pm 0,58)$ dos grupos controle e teste, respectivamente, não apresentaram diferença significativa ao final do experimento. Entretanto, a concentração de lipídio total sofreu uma redução significativa $(p<0,05)$ ao longo do estudo, em ambos os grupos, sendo mais pronunciada no grupo controle $(74 \%)$, estando ao final do experimento o teor de lipídio mais elevado $(\rho<0,05)$ no grupo teste $(1,87 \pm 0,03)$ do que no grupo controle $(1,12 \pm 0,021)$. Contudo, o teor de colesterol no grupo teste $(0,115 \pm 0,004)$ apresentou-se inferior ao do grupo controle $(0,1306 \pm 0,001)$, embora sem diferença estatística significativa. Conclui-se que a semente de linhaça na ração parece aumentar o lipídio dos camarões, sugerindo que esse aumento não implica em elevação do colesterol. Havendo, ainda, a possibilidade de ser devido à maior incorporação dos ácidos graxos poliinsaturados.

Palavras-chave: camarão; macronutrientes; linhaça; colesterol.
\end{abstract}

\begin{abstract}
The aim of this study was to analyze the chemical composition of shrimp fed with a diet complemented with linseed. 800 post larvae shrimp were divided into two types of treatment: 1) a test group based on casein complemented with linseed, and 2) a casein group. At the end of the experiment, the two treatments showed no significant differences in the mean values of humidity $(79.8 \pm 0.82 ; 78.9 \pm 1.68)$, ashes $(0.62 \pm 0.07 ; 0.68 \pm 0.2)$, carbohydrates $(2.63 \pm 0.13 ; 1.58 \pm 0.7)$ and proteins $(15.6 \pm 1.02 ; 16.0 \pm 0.58)$. However, the concentration of total lipids in both groups declined significantly during this study. This decline was more marked in the control group (74\%). At the end of the experiment, the lipid content was higher $(p<0.05)$ in the test group $(1.87 \pm 0.03)$ than in the control group $(1.12 \pm 0.021)$. However, the cholesterol content in the test group $(0.115 \pm 0.004)$ was lower than in the control group $(0.1306 \pm 0.001)$, albeit without a statistically significant difference. It was concluded that the addition of linseed to the food seems to increase the shrimps' lipids, which suggests that this increase does not imply heightened cholesterol levels. It is also possible that the increase in lipids is due to the incorporation of a large proportion polyunsaturated fatty acids.

Keywords: shrimp; macronutrients; linseed; cholesterol.
\end{abstract}

\section{Introdução}

Os camarões de água doce do gênero Macrobrachium possuem boa aceitabilidade no mercado consumidor, fato que tem estimulado a exploração de estoques naturais ${ }^{26}$. Por isso, o cultivo deste tipo de camarão é um dos setores da aqüicultura que mais cresce, em termos gerais. Segundo a $\mathrm{FAO}^{8}$, entre 1990 e 2000, a produção de Macrobrachium rosenbergii passou de 21.000 para 118.500 toneladas, com quase $500 \%$ de aumento. No Brasil, a produção de camarões cresceu significativamente nos últimos anos e além de aumentar a capacidade de suprir a demanda do mercado interno e apresentar excelentes condições para expansão, o potencial para exportação também vem aumentando ${ }^{26}$.

São vários os fatores que determinam a composição química de uma espécie de peixe. Dentre eles, podemos citar a

Recebido para publicação em 19/1/2007

Aceito para publicação em 24/7/2007 (002166)

Faculdade de Nutrição, Universidade Federal Fluminense - UFF

Rua São Paulo, 30, $4^{0}$ andar, Centro, Niterói - RJ, Brasil

E-mail: vilma.blondet@predialnet.com.br

2 Faculdade Farmácia, Universidade Federal Fluminense - UFF

Rua Mário Viana, 523, Santa Rosa, CEP 24241-000, Niterói - RJ, Brasil

*A quem a correspondência deve ser enviada alimentação, genética, sexo, tipo e época da desova, estágio produtivo, tamanho e migração, além de fatores ambientais relacionados com variações em seu habitat e formas de criação $0^{1,15,18,34}$. A alimentação é considerada um dos principais fatores determinantes da composição do tecido muscular e, especialmente, da composição de ácidos graxos dos lipídios deste tecido.

Entretanto, pouca informação sobre a composição química do camarão produzido no Brasil está disponível. Sabe-se que o músculo do camarão, assim como o de todo o pescado, em geral, é composto de proteínas de elevado valor nutritivo por conter alta proporção de aminoácidos essenciais, expressivo teor de minerais ${ }^{21}$ e baixa quantidade de lipídios totais ${ }^{7}$. Os alimentos de origem animal são, a rigor, as principais fontes de colesterol na dieta, estando intimamente associados aos lipídios totais. Sabe-se que o camarão é um fruto do mar considerado de alto conteúdo de colesterol, contudo sua concentração em ácido graxo poliinsaturado (AGPI) é também considerada elevada, e existe a possibilidade de que a alta concentração de AGPI possa anular os efeitos nocivos do colesterol no organismo humano ${ }^{7}$. 
Em decorrência dos efeitos aterogênicos causados pelo consumo excessivo de ácidos graxos saturados e colesterol pela população, iniciaram-se diversas campanhas de educação nutricional, sugerindo mudanças de hábito alimentar, preconizando a substituição de grande parte dos ácidos graxos saturados da dieta por AGPI. Este fato vem estimulando a realização de pesquisas com animais, como por exemplo: aves de postura, visando reduzir os teores de ácidos graxos saturados da gema do ovo ${ }^{9}$.

Alguns estudos foram realizados com diferentes animais, incluindo o peixe, como a tilápia do $\mathrm{Nilo}^{14,30,31}$, o escargot francês ${ }^{13}$, aves poedeiras ${ }^{12}$ e em mamíferos ${ }^{29,33}$, com o objetivo de alterar a composição dos ácidos graxos em seu organismo através da modificação de rações, visando obter produto final com teor elevado de ácidos graxos essenciais, principalmente da série $\omega-3$ e $\omega-6^{13}$.

De acordo com o conhecimento científico, a linhaça é considerada um alimento funcional com boa concentração de proteínas, apresentando ainda propriedades antiaterogênicas devido ao seu alto conteúdo de lipídios insaturados, principalmente de $\omega-3^{23,24}$. Assim, o presente estudo objetiva analisar o efeito do consumo de ração adicionada de semente de linhaça sobre a concentração de macronutrientes, e de colesterol, no camarão de água doce (Macrobrachium rosenbergii), por considerar de grande interesse e importância a realização de estudos relacionados à melhoria da qualidade nutricional de alimentos que possam ser produzidos, comercializados e introduzidos na alimentação da população sem riscos para a saúde.

\section{Material e métodos}

O experimento foi realizado em Itaipuaçu, município de Maricá, no Estado do Rio de Janeiro. Foram utilizadas 800 póslarvas de camarão d'água doce (Macrobrachium rosenbergii) oriundos da Fazenda Santa Helena, Silva Jardim (RJ). As pós-larvas foram divididas em dois grupos: 1) grupo controle: alimentado com ração à base de caseína; e 2) grupo testealimentado com ração à base de caseína adicionada de linhaça. Ambos foram estocados em tanques de cimento tampados com telas transparentes para evitar entrada de predadores. Cada tanque recebeu oxigenação constante, por meio de bomba elétrica (marca Sarlo Better mod. S300 - 110 v com capacidade de 280 L/h) e pingadeiras que auxiliaram na oxigenação e renovação da água. A limpeza dos tanques foi realizada duas vezes por semana, com renovação de $80 \%$ do volume da água.

A ração foi fornecida 2 vezes ao dia, sendo a quantidade administrada na $1^{\mathrm{a}}$ quinzena de $0,1 \mathrm{~g}$ de ração por camarão, e nos dias posteriores o equivalente a $5 \%$ da biomassa total dos viveiros, de forma equivalente para os dois tratamentos ${ }^{28}$. Quinzenalmente, os camarões foram pesados em grupo. Para obtenção do peso de cada pós-larva, o peso total do grupo foi dividido pelo número de camarões e assim obtido o peso médio.

\subsection{Preparo das rações}

As rações foram balanceadas para o animal em estudo segundo as características bromatológicas do manual de Carcinicultura de água doce ${ }^{28}$ (Quadro 1). Os ingredientes utilizados no preparo da ração do grupo teste e controle são apresentados na Tabela 1. Estes foram homogeneizados por 10 minutos em batedeira industrial, e posteriormente foi acrescentada água fervente aos poucos para gelatinizar o amido e formar uma massa com textura lisa. Após dar o ponto à massa, esta foi colocada no peletizador para formação de pequenos peletes, arrumados em tabuleiros e colocados em estufa ventilada a $51{ }^{\circ} \mathrm{C}$ por 22 horas, até obtenção de umidade ao redor de $8 \%$.

Para a manipulação da ração do grupo teste, a semente de linhaça foi triturada em um moinho para obtenção de uma farinha fina e homogênea. Devido à composição química da semente de linhaça (Quadro 2) já conter óleo e fibras em quantidades adequadas, não foi preciso adicionar esses nutrientes à ração teste. As rações, dessa forma, mantiveram-se isoprotéica (teste $=29,8 \mathrm{~g}$; controle $=29,7 \mathrm{~g}$ ) e isocalórica (teste $=334,8 \mathrm{kcal}$; controle $=316,5 \mathrm{kcal})$.

Quadro 1. Características bromatológicas ideais para ração do camarão da Malásia.

\begin{tabular}{|lr|}
\hline Proteína bruta & entre $25-30 \%$ \\
Carboidratos (açúcares) & entre $30-40 \%$ \\
Lipídios & entre $6-8 \%$ \\
Fibras & entre $6-8 \%$ \\
Outros (cinzas) & entre $8-10 \%$ \\
Umidade & até $10 \%$ \\
Relação $\mathrm{Ca} / \mathrm{P}$ & entre $2,5 / 1 \%$ \\
\hline
\end{tabular}

Referência: SEBRAE/ES, 2005.

Quadro 2. Composição química da semente de linhaça utilizada no experimento (100 g).

\begin{tabular}{|lc|}
\hline Valor calórico & $470 \mathrm{kcal}$ \\
Carboidrato & $30 \mathrm{~g}$ \\
Proteína & $20 \mathrm{~g}$ \\
Lipídios totais & $30 \mathrm{~g}$ \\
Fibra alimentar & $30 \mathrm{~g}$ \\
\hline
\end{tabular}

Tabela 1. Formulação das diferentes rações utilizadas no experimento (100 g).

\begin{tabular}{|c|c|c|}
\hline Ingredientes & $\begin{array}{c}\text { Ração a base de } \\
\text { caseína }+ \text { linhaça }\end{array}$ & $\begin{array}{c}\text { Ração a base de } \\
\text { caseína }\end{array}$ \\
\hline Caseína $^{1}$ & 31,66 & 38,09 \\
\hline Semente de linhaça & 25,37 & - \\
\hline Amido & 35 & 38,85 \\
\hline Óleo de Soja & - & 7,48 \\
\hline Celulose & - & 7,61 \\
\hline $\mathrm{BHT}^{2}$ & 0,02 & 0,02 \\
\hline Calcário & 2,10 & 2,10 \\
\hline Fosfato bicálcico & 5,30 & 5,30 \\
\hline $\begin{array}{l}\text { Suplemento mineral } \\
\text { e vitamínico }\end{array}$ & 0,5 & 0,5 \\
\hline Vitamina $\mathrm{C}^{4}$ & 0,05 & 0,05 \\
\hline Total & 100 & 100 \\
\hline \multicolumn{3}{|c|}{$\begin{array}{l}{ }^{1} \text { Caseína comercial Rhoster Industria e Comércio Ltda ( } 78 \% \text { de proteína); }{ }^{2} \text { Butil-hidroxi- } \\
\text { tolueno (antioxidante); }{ }^{3} \text { Níveis de garantia por kg de premix (Suprevit - SUPREMAIS): vit. } \\
\text { A } 760.000 \text { UI; vit. D3 } 380.000 U I \text {; vit. E } 28.000 \mathrm{mg} \text {; vit. K3 } 4.000 \mathrm{mg} \text {; vit. B1 } 3.000 \mathrm{mg} \text {; vit. } \\
\text { B2 } 4.000 \mathrm{mg} \text {; niacina } 26.000 \mathrm{mg} \text {; vit. B6 } 4.000 \mathrm{mg} \text {; vit. B12 } 4.000 \mathrm{mg} \text {; ácido pantotênico } \\
8.000 \mathrm{mg} \text {; ácido fólico } 1.400 \mathrm{mg} \text {;it. C } 30.000 \mathrm{mg} \text {; biotina } 40 \mathrm{mg} \text {; colina } 168 \mathrm{~g} \text {; metionina } \\
100 \text { g; cobre } 10.000 \mathrm{mg} \text {; iodo } 60 \mathrm{mg} \text {; manganês } 2.000 \mathrm{mg} \text { selênio } 30 \mathrm{mg} \text {; zinco } 20.000 \mathrm{mg} \text {; } \\
\text { antifúngico } 200 \text { g; antioxidante } 30 \text { g; e }{ }^{4} 2 \text {-monofosfato de ácido ascórbico L. }\end{array}$} \\
\hline
\end{tabular}




\subsection{Determinação da composiçáo centesimal}

As análises químicas dos camarões em diferentes fases de crescimento foram realizadas em triplicatas, no Laboratório de Bromatologia da faculdade de Farmácia da Universidade Federal Fluminense (UFF). Devido ao tamanho dos camarões, as análises foram feitas de corpo inteiro com casca e cabeça.

No primeiro dia de experimento, e a cada 15 dias, foram coletados aproximadamente $10 \mathrm{~g}$ de camarão de cada grupo. Estas amostras foram acondicionadas em embalagem apropriada e congeladas a $-20{ }^{\circ} \mathrm{C}$ para posteriores análises até o final do experimento, aos 60 dias. A determinação de umidade residual foi realizada segundo as Normas Analíticas do Instituto Adolfo Lutz ${ }^{16}$; proteína pelo método de microkjeldahl, segundo a $\mathrm{AOAC}^{4}$; cinzas por incineração em mufla a $550{ }^{\circ} \mathrm{C}$, segundo as Normas Analíticas do Instituto Adolfo Lutz ${ }^{16}$; a extração de lipídio total foi determinada pelo método de Bligh-Dyer ${ }^{5} \mathrm{e}$ a determinação quantitativa foi realizada gravimetricamente. NIFEXT (Nitrogen-free extract) realizada através da diferença entre 100 e o somatório das determinações de umidade, gordura, proteína e cinzas, obtendo-se assim o percentual médio de carboidrato ${ }^{19}$. E o teor de colesterol foi determinado através do kit para análise de Colesterol Liquiform (Labtest), segundo metodologia descrita por BRAGAGNOLO et al. ${ }^{6}$.

\subsection{Análise estatística}

Os resultados são apresentados através da estatística descritiva, como média e desvio padrão. Utilizamos o teste $t$ de student não-pareado para comparação entre as médias dos dois grupos e o teste t de student pareado para analisar as médias dos grupos em diferentes momentos (dias 15, 30, 45 e 60). Foi aceito um nível de significância de $5 \%$. As análises foram realizadas através do programa GraphPad InStat.

\section{Resultados e discussão}

A Tabela 2 mostra a composição química dos camarões submetidos aos diferentes tratamentos. Os valores médios de umidade, cinzas, carboidratos e proteínas não apresentaram diferença significativa entre os tratamentos, ao final do experimento. Contudo, observa-se que o grupo teste apresentou as maiores médias, ao longo do experimento, para proteína e cinzas, enquanto o grupo controle apresentou maior concentração de carboidratos $(\mathrm{p}<0,05)$ no $30^{\circ}$ dia de ensaio.

Os valores médios de umidade encontrados neste experimento variaram muito pouco ao longo do estudo e entre os grupos, estando próximos aos valores relatados pelo IBGE (79,2 g. $\left.100 \mathrm{~g}^{-1}\right)^{11}$ e dos valores encontrados por KIRSCHNIK e $\operatorname{VIEGAS}^{17}\left(78,54\right.$ g.100 $\left.\mathrm{g}^{-1}\right)$ em camarões Macrobrachium rosenbergii, contudo, inferiores ao relatados por PEDROSA e COZZOLINO $^{21}\left(88,34\right.$ g. $\left.100 \mathrm{~g}^{-1}\right)$ em camarões Penaeus brasiliensis.

O teor de cinzas do grupo teste $(0,68 \pm 0,22)$ encontra-se similar ao do grupo controle $(0,62 \pm 0,07)$, ao final do experimento; apresentando um aumento a partir do valor inicial de $60 \%$ no grupo controle e de $75 \%$ no grupo teste (Tabela 3 ). Em ambos os grupos, a concentração de cinzas encontra-se inferior aos resultados obtidos por PEDROSA e COZZOLINO ${ }^{21}$ (1,05 g.100 g ${ }^{-1}$ ) em camarões Penaeus brasiliensis, por KIRSCHNIK e VIEGAS $^{17}$ (1,35 g. $100 \mathrm{~g}^{-1}$ ) em Macrobrachium rosenbergii, e pelo $\operatorname{IBGE}^{11}\left(1,4 \mathrm{~g}^{1} 100 \mathrm{~g}^{-1}\right)$.

Pode-se observar um incremento na concentração de proteína ao longo do estudo, ao redor de $9 \%$ para o grupo controle e de $12 \%$ para o grupo teste, no final do experimento. Entretanto, a concentração média de proteína, ao final do estudo, apresenta-se similar entre os grupos estudados (Tabela 2), e superior ao citado por PEDROSA e COZZOLINO ${ }^{21}$ (10,62 g.100 $\mathrm{g}^{-1}$ ). Contudo, inferior aos valores reportados por KIRSCHNIK e $\operatorname{VIEGAS}^{17}$ (19,50 g.100 g $\left.{ }^{-1}\right)$, pelo $\operatorname{IBGE}^{11}\left(17,6\right.$ g. $\left.100 \mathrm{~g}^{-1}\right)$, e por $\mathrm{FRANCO}^{10}\left(20,6 \mathrm{~g} .100 \mathrm{~g}^{-1}\right)$. Estes resultados mostram que o camarão Macrobrachium rosenbergii é uma rica fonte de proteína para a dieta humana.

A concentração média de carboidrato do grupo controle apresentou-se $30 \%$ superior a do grupo teste, ao final do experimento. Contudo, estes resultados não mostraram diferença significativa (Tabela 2). A concentração de carboidrato, em ambos os grupos, apresentou-se superior à relatada na literatura por FRANCO $^{10}$, PINHEIRO ${ }^{22}$ e IBGE ${ }^{11}$.

A concentração de lipídios totais e de colesterol é apresentada na Tabela 3. Pode-se observar uma diminuição significativa dos níveis de lipídios totais (acima de 50\%) mais pronunciada no grupo controle, ao longo do estudo. Os resultados sugerem que a utilização da semente de linhaça como um complemento alimentar na ração do camarão parece exercer influência sobre os lipídios, pois mesmo apresentando uma redução do teor inicial de gordura ao longo do experimento, o grupo controle sempre teve significativamente $(\mathrm{p}<0,05)$ menor teor de lipídios que o grupo teste. Estudos realizados com o camarão da Malásia mostram níveis mais baixos de lipídios totais ${ }^{10,11,17,21,22}$ (0,20 g. $100 \mathrm{~g}^{-1} ; 0,36$ g. $100 \mathrm{~g}^{-1} ; 0,90$ g. $100 \mathrm{~g}^{-1} ; 0,37$ g. $100 \mathrm{~g}^{-1}$; $0,9 \mathrm{~g} .100 \mathrm{~g}^{-1}$ ) do que os encontrados no presente estudo para ambos os grupos, controle e teste.

Embora os níveis médios de colesterol não se apresentem estatisticamente diferentes entre os grupos, pode-se observar

Tabela 2. Composição físico-química dos camarões (Macrobrachium rosenbergii) submetidos aos diferentes tratamentos (g. 100 g ${ }^{-1}$ ).

\begin{tabular}{|c|c|c|c|c|c|c|c|c|c|c|}
\hline \multirow[t]{2}{*}{ Composição } & \multicolumn{2}{|c|}{ Dia 0} & \multicolumn{2}{|c|}{ Dia 15} & \multicolumn{2}{|c|}{ Dia 30} & \multicolumn{2}{|c|}{ Dia 45} & \multicolumn{2}{|c|}{ Dia 60} \\
\hline & G C & G T & $\mathrm{G} \mathrm{C}$ & G T & G C & G T & G C & G T & G C & G T \\
\hline Umidade & $81,0 \pm 4,6$ & $81,0 \pm 4,6$ & $75,7 \pm 0,28$ & $73,1 \pm 0,8$ & $77,6 \pm 0,43^{*}$ & $74,4 \pm 0,74$ & $77,3 \pm 1,92$ & $75,2 \pm 2,21$ & $80,0 \pm 0,82$ & $79,5 \pm 1,0$ \\
\hline Cinzas & $0,39 \pm 0,15$ & $0,39 \pm 0,15$ & $0,87 \pm 0,04$ & $0,92 \pm 0,07$ & $0,86 \pm 0,06$ & $1,01 \pm 0,17$ & $0,81 \pm 0,11$ & $1,02 \pm 0,08^{*}$ & $0,62 \pm 0,07$ & $0,68 \pm 0,22$ \\
\hline Proteína & $14,3 \pm 4,44$ & $14,3 \pm 4,44$ & $17,4 \pm 1,53$ & $19,7 \pm 2,53$ & $15,9 \pm 0,12$ & $19,4 \pm 0,77^{*}$ & $16,2 \pm 1,94$ & $18,9 \pm 1,75$ & $15,6 \pm 1,02$ & $16,0 \pm 0,58$ \\
\hline Carboidrato & $0,05 \pm 0,00$ & $0,05 \pm 0,00$ & $2,13 \pm 0,2$ & $2.25 \pm 0,2$ & $3,84 \pm 0,11$ & $3,04 \pm 0,18^{*}$ & $4,42 \pm 0,74$ & $2,33 \pm 0,65$ & $2,66 \pm 0,13$ & $1,95 \pm 0,55$ \\
\hline
\end{tabular}


Tabela 3. Teores médios de lipídeos totais e colesterol no tecido de camarões Macrobrachium rosenbergii alimentados com diferentes rações (g).

\begin{tabular}{ccc}
\hline \multicolumn{1}{c}{ Dias } & Lipídeo total & \multicolumn{1}{c}{ Colesterol } \\
\hline Dia 0 & & \\
Grupo C & $4,26 \pm 0,01$ & $0,1554 \pm 0,004$ \\
Grupo T & $4,26 \pm 0,01$ & $0,1554 \pm 0,004$ \\
Dia 15 & & \\
$\quad$ Grupo C & $3,9 \pm 0,16$ & $0,1719 \pm 0,001$ \\
$\quad$ Grupo T & $4,03 \pm 0,00$ & $0,1591 \pm 0,000$ \\
Dia 30 & & \\
$\quad$ Grupo C & $1,80 \pm 0,06$ & $0,174 \pm 0,006$ \\
$\quad$ Grupo T & $2,15 \pm 0,25$ & $0,1605 \pm 0,006$ \\
Dia 45 & $1,27 \pm 0,007^{\mathrm{a}}$ & $0,1227 \pm 0,009$ \\
$\quad$ Grupo C & $2,55 \pm 0,05^{\mathrm{b}}$ & $0,1102 \pm 0,004$ \\
$\quad$ Grupo T & & \\
Dia 60 & $1,12 \pm 0,021^{\mathrm{a}}$ & $0,1306 \pm 0,001$ \\
$\quad$ Grupo C & $1,87 \pm 0,03^{\mathrm{b}}$ & $0,1157 \pm 0,004$ \\
Grupo T &
\end{tabular}

Grupo $\mathrm{C}$ = grupo controle; grupo $\mathrm{T}=$ grupo teste; os valores são apresentados como média \pm desvio padrão; e diferentes letras sobrescritas denotam diferença estatisticamente significativa $(p>0,05)$ entre os grupos.

que o grupo controle apresentou as maiores médias ao longo do estudo. Foi observada uma redução maior do teor de colesterol no grupo teste $(-25,6 \%)$ em comparação ao grupo controle $(-15,96 \%)$, ao final do experimento (Tabela 3$)$. Também foi observado, que para ambos os grupos - até 30 dias de tratamento - o teor de colesterol apresentou-se superior ao relatado por BRAGAGNOLO e AMAYA ${ }^{7}$ (139 mg. $100 \mathrm{~g} \mathrm{~g}^{-1}$ ), enquanto os resultados de 45 e 60 dias (em ambos os grupos) apresentaram valores inferiores ao citado por estes mesmos autores. Estes resultados corroboram o estudo realizado por VISENTAINER et al. ${ }^{32}$, que mostraram que o fornecimento de óleo de linhaça até um nível de 3,75\% parece baixar as concentrações de colesterol no filé de tilápias. Segundo BRAGAGNOLO e RODRIGUEZ-AMAYA ${ }^{7}$, em termos de saúde humana, o alto conteúdo de colesterol do camarão é compensado pelo baixo conteúdo total de lipídio e a predominância de AGPI, especialmente $\omega$-3. Além disso, os resultados de OLIVEIRA et al. ${ }^{20}$ mostraram que em indivíduos normolipidêmicos foi observado que o consumo moderado de camarão não afetou adversamente o perfil total de lipoproteínas.

A redução do teor de gordura e a adição de semente de linhaça podem estar contribuindo para a redução do teor de colesterol, visto que houve uma redução deste composto ao longo do experimento, em ambos os grupos, sendo mais pronunciada no grupo teste, devido à possibilidade de maior incorporação dos AGPI (presentes na linhaça) nos tecidos do camarão Macrobrachium rosenbergii.

A Tabela 4 mostra a média de variação do peso do Macrobrachium rosenbergii submetido aos diferentes tratamentos. Podem ser observados valores superiores de peso no grupo teste em relação ao grupo controle a partir dos 30 dias; contudo esta diferença não apresenta-se estatisticamente significativa $(\mathrm{p}>0,05)$. O peso médio final dos camarões do grupo teste, no presente estudo, apresentou-se próximo ao citado por SAMPAIO et al. ${ }^{27}$ em Macrobrachium amazonicum e aos valores encontrados por QUERIJERO et al.$^{25}$, porém superior aos valores relatados por ARAÚJO e VALENTI ${ }^{3}$ e ARAÚJO e PORTZ ${ }^{2}$. Enquanto a média de peso obtida aos 60 dias de experimento do grupo controle apresentou-se inferior aos valores citados por SAMPAIO et al. ${ }^{27}$ e QUERIJERO et al. ${ }^{25}$, os valores relatados por ARAÚJO e VALENTI ${ }^{3}$ e ARAÚJO e PORTZ ${ }^{2}$ apresentaram-se superiores.

Tabela 4. Peso médio e ganho de peso do Macrobrachium rosenbergii nos diferentes tratamentos.

\begin{tabular}{ccc}
\hline & Peso (mg) & Ganho de peso (mg) \\
\hline Dia 0 & 100 & 0 \\
Grupo C & 100 & 0 \\
Grupo T & & \\
Dia 15 & 148 & 48 \\
Grupo C & 146 & 46 \\
Grupo T & & \\
Dia 30 & 176 & 76 \\
Grupo C & 239 & 139 \\
Grupo T & & \\
Dia 45 & 225 & 125 \\
Grupo C & 330 & 230 \\
Grupo T & & 130 \\
Dia 60 & 230 & 290 \\
Grupo C & 390 & \\
Grupo T & &
\end{tabular}

\section{Conclusão}

A utilização da semente de linhaça como complemento na ração de camarão da Malásia parece influenciar a concentração de lipídios totais, sem, no entanto, elevar a concentração de colesterol. Não havendo influência também sobre os teores de umidade, cinzas, carboidratos e proteínas.

\section{Referências bibliográficas}

1. AHLGREN, G. et al. Fatty acid content of dorsol muscle-a indicator of fat quality in freshwater fish. Journal of Fish Biology, v. 45 , p. 131-57, 1994.

2. ARAÚJO, FRANCISCO G.; PORTZ, L. Consorciamento do camarão gigante da Malásia (Macrobrachium rosenbergii) com tilápia nilótica (Oreochrmis niloticus) e com carpa comum (Cyprinus carpio) em diferentes taxas de estocagem. Boletim Instituto Pesca. v. 24, n. especial, p. 57-69, 1997.

3. ARAÚJO, M. C.; VALENTI, W. C. Manejo alimentar de pós-larvas do camarão da Amazônia, Macrobrachium amazonicum, em berçário I. Acta Scientiarum Animal Sciences. Maringá, v. 27, n. 1, p. 67-72, 2005.

4. ASSOCIATION OF OFFICIAL ANALYTICAL CHEMISTS. Official Methods of Analysis. Arlington, AOAC, 1995.

5. BLIGH, E.G.; DYER, W. J. A rapid method of total lipid extraction and purification. Canadian Journal of Biochemistry, v. 37, p. 911-917, 1959.

6. BRAGAGNOLO, N.; RODRIGUEZ-AMAYA, D. B. Determinação de colesterol em carne: comparação de um método colorimétrico e um método por cromatografia líquida de alta eficiência. Revista do Instituto Adolf Lutz, v. 60, n. 1, p. 53-57, 2001. 
7. BRAGAGNOLO, N.; RODRIGUEZ-AMAYA, D. B. Total lipid, cholesterol, and fatty acids of farmed freshwater prawn (Macrobrachium rosenbergii) and wild marine shrimp (Penaeus brasiliensis, Penaeus schimitti, Xiphopenaeus kroyeri); Journal of Food Composition and Analysis, v. 14, p. 359-369, 2001.

8. FAO. Food and Agriculture Organization of the United Nations. Yearbook of fishery statistics: summary table. FAO, Roma (http:/ www.fao.org) 2002.

9. FARIAS FILHO, R. V. Efeito do óleo de linhaça e vitamina E na dieta de poedeiras cobre o perfil de ácidos graxos da gema. Maringá, 2000. 47 f. Dissertação (Mestrado em Zootecnia) Programa de Pós-graduação em Zootecnia, Universidade Estadual de Maringá, 2000.

10. FRANCO, G. Tabela de composição química dos alimentos. $9^{a}$ edição, Rio de Janeiro. Editora Atheneu, p 117, 1992.

11. FUNDAÇÃO INSTITUTO BRASILEIRO DE GEOGRAFIA E ESTATÍSTICA. Estudo nacional da despesa familiar: tabela de composição dos alimentos. Secretaria de Planejamento da Presidência da República. Rio de Janeiro, v. 3, p. 82, 1997.

12. GÓMEZ, M. E. D. B. Modulação da composição de ácidos graxos poliinsaturados ômega 3 de ovos e tecidos de galinhas poedeiras, através da dieta. I Estabilidade oxidativa. São Paulo, 2003.149 p. Tese (doutorado em Bromatologia) - Universidade de São Paulo, 2003.

13. HAYASHI, C. et al. Desempenho e características de carcaça do escargot francês (Helix aspersa máxima) alimentados com rações contendo diferentes óleos vegetais. Ciência Rural v. 34, n. 1, Santa Maria, Jan/Fev. 2004.

14. HAYASHI, C. et al. Uso de diferentes óleos vegetais em dietas para a tilápia do Nilo (Oreochromis niloticus, L.), na fase inicial. In: REUNIÃO ANUAL DA SOCIEDADE BRASILEIRA DE ZOOTECNIA, 37, 2000, Porto Alegre. Anais..., Porto Alegre: SBZ, 2000a. Disponível na Internet: http //www. sbz.org.Br /scripts/ anais 2000/index.asp

15. HENDERSON, R. J.; TOCHER, D. R. The lipid composition and biochemistry of freshwater fish. Progress in Lipid Research, v. 26, p. 281-347, 1987.

16. INSTITUTO ADOLFO LUTZ. Normas analíticas do Instituto Adolfo Lutz: métodos químicos e físicos para análise de alimentos. $3^{\text {a }}$ ed. São Paulo: IAL, v.1, p.16-75, 245-246, 1985.

17. KIRSCHNIK, P. G.; VIEGAS, E. M. M. Alterações na qualidade do camarão de água doce Macrobrachium rosenbergii durante estocagem em gelo. Ciência e Tecnologia de Alimentos, Campinas, v. 24, n. 3, p. 407-412, 2004.

18. MOREIRA, A. B. et al. Fatty acids profile and cholesterol contents of three Brazillian brycon freshwater fishes. Journal of Food Composition and Analysis, v. 14, p. 565-74, 2001.

19. OLIVEIRA, E. C. M. et al. Composição centesimal do cogumelo do sol (Agaricus blazei). Revista Universidade Alfenas, Alfenas, v. 5 , p. 169-172,1999.

20. OLIVEIRA, E. et al. Efects of shrimp consumption on plasma lipoproteins. American Journal of Clinical Nutrition. v. 64, p. 712-717, 1996.
21. PEDROSA, L. F. C.; COZZOLINO, S. M. F. Composição centesimal e de minerais de mariscos crus e cozidos da cidade de Natal/ RN. Ciência e Tecnologia de Alimentos, v. 21, n. 2, Campinas, maio-agosto, 2001.

22. PINHEIRO, A. B. V. Tabela para avaliação de consumo alimentar em medidas caseiras. $3^{\circ}$ ed. Rio de Janeiro, Diagramação e editoração eletrônica, p. 21, 1996.

23. PRASAD K. Dietary flax seed in prevention of hypercholesterolemic atherosclerosis. Atherosclerosis, Shannon, v. 132, p. 69-76, 1997.

24. PRASAD, K.; MANTHA, S. V.; MUIR, A. D.; WESTCOTT, N. D. Reduction of hypercholesterolemic atherosclerosis by CDCflaxseed with very low alpha-linolenic acid. Atherosclerosis, Shannon, v. 136, p. 367-375, 1998.

25. QUERIJERO, B. V. L. et al. Utilization of monounsaturated fatty acid (18: 1n-9, oleic acid) by freshwater prawn Macrobrachium rosenbergii ( de Man) juveniles. Aquaculture Nutrition, n. 3, p. 127-139, 1997.

26. REIS, J. A. et al. Estudo higiênico-sanitário dos camarões dulcícolas Macrobrachium amazonicum e M. Jelskil. Higiene Alimentar, São Paulo, v. 18, n. 116-117, p. 58-67, 2004.

27. SAMPAIO, F. G. et al. Níveis de vitamina $\mathrm{E}$ e de selênio para póslarvas de Macrobrachium amazonicum. Scientiarum. Animal Sciences. Maringá, v. 26, n.1, p. 129-135, 2004.

28. SEBRAE/ES. 2005. Serviço de apoio às micro e pequenas empresas do Espírito Santo. Tecnologia de criação do camarão da Malásia (Macrobrachium rosenbergii) Manual de Carcinicultura de Água Doce. Centro de Tecnologia em Aqüicultura e Meio Ambiente LTDA- CTA, Vitória, 2005.

29. SOUZA, R. V. et al. Desempenho e Características de carcaça de suínos de 70 a $100 \mathrm{~kg}$ alimentadas com rações contendo diferentes óleos.Ciências agrotécnica. Lavras. Edição Especial, p. 1679-1684, dez, 2003.

30. VISENTAINER, J. V. et al. Efeito do tempo de fornecimento de ração suplementada com óleo de linhaça sobre a composição físico-química e de ácidos graxos em cabeças de tilápias do Nilo (Oreochromis niloticus). Ciência e tecnologia de Alimentos, Campinas, v. 23, n. 3. p. 478-484,2003.

31. VISENTAINER, J. V. et al. Quantificação de ácidos graxos ômega-3 (LNA, EPA e DHA), caracterização físico-química e composição de ácidos graxos em cabeças de tilápias jovens. In: CONGRESSO BRASILEIRO DE CIÊNCIA E TECNOLOGIA DE ALIMENTOS, 17, 2000, Fortaleza. Resumos... Fortaleza: UFC/SBCTA, p. 5221, 2000.

32. VISENTAINER, J. V. et al. Relação entre teores de colesterol em filés de tilápias e níveis de óleo de linhaça na ração. Ciência e Tecnologia de Alimentos. v. 25, n. 2, 2005.

33. WEBSTER, N. K. et al. Modulation of brain lipids of rats by various dietary oils: sunflower, high-oleic sunflower, olive, rapeseed and coriander oil. Nutrition Research, v. 19, n. 7, p. 997-1007, 1999.

34. ZENEBE, T.; AHLGREN, G.; GUSTAFSSON, I. B. Fatty acid and lipid content or Oreochromis niloticus L. in Ethiopian Lakes - dietary effects of phytoplankton. Ecology of Freshwater Fish. Journal of Fish Biology, v. 7, p. 146-58, 1998. 